\title{
STATIONARY SOLUTIONS OF TWO-DIMENSIONAL HETEROGENEOUS ENERGY MODELS WITH MULTIPLE SPECIES
}

\author{
ANNEGRET GLITZKY and ROLF HÜNLICH \\ Weierstrass Institute for Applied Analysis and Stochastics \\ Mohrenstr. 39, D-10117 Berlin, Germany \\ E-mail: glitzky@wias-berlin.de, huenlich@wias-berlin.de
}

\begin{abstract}
We investigate stationary energy models in heterostructures consisting of continuity equations for all involved species, of a Poisson equation for the electrostatic potential and of an energy balance equation. The resulting strongly coupled system of elliptic differential equations has to be supplemented by mixed boundary conditions.

If the boundary data are compatible with thermodynamic equilibrium then there exists a unique steady state. We prove that in a suitable neighbourhood of such a thermodynamic equilibrium there exists a unique steady state, too. Our proof is based on the Implicit Function Theorem and on regularity results for systems of strongly coupled elliptic differential equations with mixed boundary conditions and non-smooth data.
\end{abstract}

\section{Introduction}

1.1. Stationary energy model for semiconductor devices. The charge transport in semiconductor devices is described by the van Roosbroeck equations (see [16]) consisting of two continuity equations for the electron and hole densities $n$ and $p$, respectively, and a Poisson equation for the electrostatic potential $\varphi$. Physical parameters occurring in these equations depend on the lattice temperature $T$ which often can be treated as a given constant parameter. This assumption is no more valid in power devices, for example. Then also the energy transport must be modelled by adding a further balance equation, and a so called energy model arises. In this paper we consider only the stationary case.

We introduce the electrochemical potential $\zeta_{n}$ of the electrons and $\zeta_{p}$ of the holes which are implicitly defined by the state equations

2000 Mathematics Subject Classification: Primary 35J55, 35A07, 35R05; Secondary 80A20.

Key words and phrases: energy models, mass, charge and energy transport in heterostructures, strongly coupled elliptic systems, mixed boundary conditions, Implicit Function Theorem, existence, uniqueness, regularity.

The paper is in final form and no version of it will be published elsewhere. 


$$
n=N F\left(\frac{\zeta_{n}+\varphi-E_{n}}{T}\right), \quad p=P F\left(\frac{\zeta_{p}-\varphi+E_{p}}{T}\right)
$$

where $N, P>0$ and $E_{n}, E_{p}$ are reference densities and reference energies, respectively. These quantities depend on $x$ and $T$. The function $F$ results from a distribution function depending on the chosen statistics. In particular we have

$$
F(y)= \begin{cases}\mathrm{e}^{y} & \text { in the case of Boltzmann statistics } \\ \mathcal{F}_{1 / 2}(y)=\frac{2}{\sqrt{\pi}} \int_{0}^{\infty} \frac{\sqrt{z} \mathrm{~d} z}{1+\exp (z-y)} & \text { in the case of Fermi-Dirac statistics. }\end{cases}
$$

The electrostatic potential fulfils the Poisson equation

$$
-\nabla \cdot(\varepsilon \nabla \varphi)=f-n+p .
$$

Here $\varepsilon>0$ is the dielectric permittivity depending on $x$, and $f$ is a given doping profile.

The remaining equations of the stationary energy model can be written in various forms. We start with the following system of differential equations consisting of two continuity equations for the electrons and holes and a conservation law for the total energy,

$$
\begin{gathered}
\nabla \cdot j_{n}=-R, \quad \nabla \cdot j_{p}=-R, \\
\nabla \cdot j_{e}=0
\end{gathered}
$$

where $R$ denotes the net recombination rate of the electron-hole generation-recombination reaction $e+h \rightleftharpoons 0$, and $j_{n}, j_{p}$ are the particle flux densities of electrons and holes, $j_{e}$ is the flux density of the total energy. Here we have to specify the underlying kinetic relations. First, we assume that the net recombination rate $R$ is given by

$$
R=r\left(\mathrm{e}^{\left(\zeta_{n}+\zeta_{p}\right) / T}-1\right)
$$

with some coefficient $r>0$. Second, we suppose that the flux densities $j_{n}, j_{p}, j_{e}$ have the form (see $[1,12])$

$$
\begin{aligned}
j_{n} & =-\left(\sigma_{n}+\sigma_{n p}\right)\left(\nabla \zeta_{n}+P_{n} \nabla T\right)-\sigma_{n p}\left(\nabla \zeta_{p}+P_{p} \nabla T\right), \\
j_{p} & =-\sigma_{n p}\left(\nabla \zeta_{n}+P_{n} \nabla T\right)-\left(\sigma_{p}+\sigma_{n p}\right)\left(\nabla \zeta_{p}+P_{p} \nabla T\right), \\
j_{e} & =-\kappa \nabla T+\sum_{i=n, p}\left(\zeta_{i}+P_{i} T\right) j_{i}
\end{aligned}
$$

with conductivities $\sigma_{n}, \sigma_{p}>0, \sigma_{n p} \geq 0, \kappa>0$, and $P_{n}, P_{p}$ are the so called transported entropies (see [11, p. 329], they are related to the thermoelectric powers of the electrons and holes, respectively). Terms containing $\sigma_{n p}$ account for some electron-hole scattering model (see [14]). All kinetic coefficients $r, \sigma_{n}, \sigma_{p}, \sigma_{n p}, \kappa, P_{n}, P_{p}$ depend on $x$ and $n, p, T$. It is important to note that the strict inequalities $r>0, \sigma_{n}, \sigma_{p}>0, \kappa>0$ are valid only for non-degenerate states $0<n, p, T<+\infty$.

The equations (2)-(4) must be supplemented by boundary conditions. The determination of these conditions is a rather complicated matter. We consider here the following version. Let $\Gamma$ be the boundary of the domain $\Omega$ which is occupied by the semiconductor device, $\nu$ the outer unit normal, and let $\Gamma_{D}$ and $\Gamma_{N}$ be disjoint, relatively open parts of 
$\Gamma$ with $\operatorname{mes}\left(\Gamma \backslash\left(\Gamma_{D} \cup \Gamma_{N}\right)\right)=0$. We require that

$$
\begin{gathered}
\zeta_{n}=\zeta_{n}^{D}, \quad \zeta_{p}=\zeta_{p}^{D}, \quad T=T^{D}, \quad \varphi=\varphi^{D} \quad \text { on } \Gamma_{D}, \\
-\nu \cdot j_{n}=g_{1}, \quad-\nu \cdot j_{p}=g_{2}, \quad-\nu \cdot j_{e}=g_{3}, \quad \nu \cdot(\varepsilon \nabla \varphi)=g_{4} \quad \text { on } \Gamma_{N} .
\end{gathered}
$$

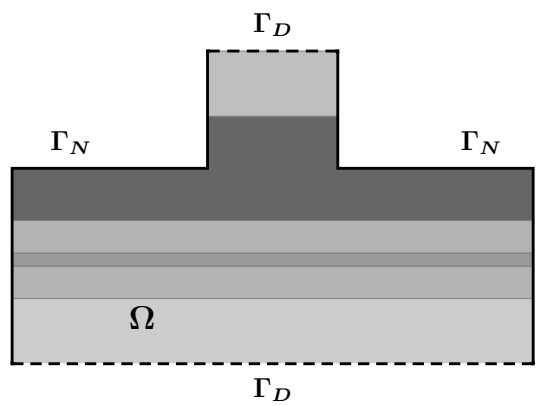

Fig. 1. Example of a heterostructure $\Omega$ consisting of different materials (highlighted by the gray shading), and the Dirichlet and Neumann parts $\Gamma_{D}$ and $\Gamma_{N}$ of the boundary which are in contact. The set $\overline{\Gamma_{D}} \cap \overline{\Gamma_{N}}$ consists of four points.

Now let us discuss some other formulations of the equations (3)-(4). Sometimes the conservation relation (4) is replaced by the heat flow equation (see also [18])

$$
-\nabla \cdot(\kappa \nabla T)=H
$$

where the right hand side

$$
\begin{aligned}
H= & -\sum_{i=n, p} \nabla \cdot\left(\left(\zeta_{i}+P_{i} T\right) j_{i}\right) \\
= & \sigma_{n}\left|\nabla \zeta_{n}+P_{n} \nabla T\right|^{2}+\sigma_{p}\left|\nabla \zeta_{p}+P_{p} \nabla T\right|^{2} \\
& \left.+\sigma_{n p} \mid \nabla\left(\zeta_{n}+\zeta_{p}\right)+\left(P_{n}+P_{p}\right) \nabla T\right)\left.\right|^{2} \\
& -T\left(\nabla P_{n} \cdot j_{n}+\nabla P_{p} \cdot j_{p}\right)+\left(\zeta_{n}+\zeta_{p}+\left(P_{n}+P_{p}\right) T\right) R
\end{aligned}
$$

contains a lot of quadratic gradient terms.

For our purpose the following reformulation is more convenient. We introduce the entropy flux density

$$
j_{s}=\frac{1}{T}\left(j_{e}-\zeta_{n} j_{n}-\zeta_{p} j_{p}\right)=-\frac{\kappa}{T} \nabla T+P_{n} j_{n}+P_{p} j_{p}
$$

For isothermal states, $\nabla T=0$, the relation $j_{s}=P_{n} j_{n}+P_{p} j_{p}$ follows which explains the meaning of $P_{n}, P_{p}$ as transported entropies. With (5) and (9) we obtain

$$
\left(\begin{array}{l}
j_{n} \\
j_{p} \\
j_{s}
\end{array}\right)=-\left(\begin{array}{lll}
\sigma_{n}+\sigma_{n p} & \sigma_{n p} & \tau_{1} \\
\sigma_{n p} & \sigma_{p}+\sigma_{n p} & \tau_{2} \\
\tau_{1} & \tau_{2} & \frac{\kappa}{T}+\tau_{3}
\end{array}\right)\left(\begin{array}{c}
\nabla \zeta_{n} \\
\nabla \zeta_{p} \\
\nabla T
\end{array}\right)
$$

where

$$
\left(\begin{array}{l}
\tau_{1} \\
\tau_{2}
\end{array}\right)=\left(\begin{array}{ll}
\sigma_{n}+\sigma_{n p} & \sigma_{n p} \\
\sigma_{n p} & \sigma_{p}+\sigma_{n p}
\end{array}\right)\left(\begin{array}{c}
P_{n} \\
P_{p}
\end{array}\right), \tau_{3}=\tau_{1} P_{n}+\tau_{2} P_{p}
$$


The matrix in (10) is symmetric and positive definite for non-degenerate states. Thus, Onsager's relations are fulfilled if we choose the fluxes $\left(j_{n}, j_{p}, j_{s}\right)$ and the generalized forces $\left(\nabla \zeta_{n}, \nabla \zeta_{p}, \nabla T\right)$. With (9), (3), (4) the entropy balance equation

$$
\nabla \cdot j_{s}=d
$$

results where $d$ is the entropy production rate,

$$
T d=-j_{n} \cdot \nabla \zeta_{n}-j_{p} \cdot \nabla \zeta_{p}-j_{s} \cdot \nabla T+R\left(\zeta_{n}+\zeta_{p}\right)
$$

Obviously $d \geq 0$, and for non-degenerate states we find that $d=0$ if and only if $\nabla \zeta_{n}=$ $\nabla \zeta_{p}=\nabla T=0, \zeta_{n}+\zeta_{p}=0$. These conditions characterize a thermodynamic equilibrium. If a thermodynamic equilibrium satisfies the boundary conditions (6), then the data in (6) necessarily fulfill the conditions

$$
\zeta_{n}^{D}=\text { const }, \quad \zeta_{p}^{D}=-\zeta_{n}^{D}, \quad T^{D}=\text { const }>0, \quad g_{1}=g_{2}=g_{3}=0 .
$$

Later on we will see that these conditions are also sufficient for the existence of a unique thermodynamic equilibrium. Corresponding equilibrium densities $n, p$ are obtained from the state equations (1) where the electrostatic potential $\varphi$ has to satisfy the nonlinear Poisson equation

$-\nabla \cdot(\varepsilon \nabla \varphi)+N\left(\cdot, T^{D}\right) F\left(\frac{\zeta_{n}^{D}+\varphi-E_{n}\left(\cdot, T^{D}\right)}{T^{D}}\right)-P\left(\cdot, T^{D}\right) F\left(\frac{\zeta_{p}^{D}-\varphi+E_{p}\left(\cdot, T^{D}\right)}{T^{D}}\right)=f$

with mixed boundary conditions

$$
\varphi=\varphi^{D} \quad \text { on } \Gamma_{D}, \quad \nu \cdot(\varepsilon \nabla \varphi)=g_{4} \quad \text { on } \Gamma_{N} .
$$

In (5) we used the fluxes $\left(j_{n}, j_{p}, j_{e}\right)$ and the generalized forces $\left(\nabla \zeta_{n}, \nabla \zeta_{p}, \nabla T\right)$. Then the Onsager relations are not valid, but they can be achieved by choosing other generalized forces, namely $\left(\nabla\left[\zeta_{n} / T\right], \nabla\left[\zeta_{p} / T\right], \nabla[-1 / T]\right)$. Then

$$
\left(\begin{array}{l}
j_{n} \\
j_{p} \\
j_{e}
\end{array}\right)=-\left(\begin{array}{lll}
\left(\sigma_{n}+\sigma_{n p}\right) T & \sigma_{n p} T & \widetilde{\tau}_{1} \\
\sigma_{n p} T & \left(\sigma_{p}+\sigma_{n p}\right) T & \widetilde{\tau}_{2} \\
\widetilde{\tau}_{1} & \widetilde{\tau}_{2} & \kappa T^{2}+\widetilde{\tau}_{3}
\end{array}\right)\left(\begin{array}{l}
\nabla\left[\zeta_{n} / T\right] \\
\nabla\left[\zeta_{p} / T\right] \\
\nabla[-1 / T]
\end{array}\right)
$$

where

$$
\left(\begin{array}{l}
\widetilde{\tau}_{1} \\
\widetilde{\tau}_{2}
\end{array}\right)=\left(\begin{array}{ll}
\left(\sigma_{n}+\sigma_{n p}\right) T & \sigma_{n p} T \\
\sigma_{n p} T & \left(\sigma_{p}+\sigma_{n p}\right) T
\end{array}\right)\left(\begin{array}{l}
\zeta_{n}+P_{n} T \\
\zeta_{p}+P_{p} T
\end{array}\right), \widetilde{\tau}_{3}=\widetilde{\tau}_{1}\left(\zeta_{n}+P_{n} T\right)+\widetilde{\tau}_{2}\left(\zeta_{p}+P_{p} T\right) .
$$

The matrix in (12) is symmetric and positive definite for non-degenerate states. The entropy production rate can be rewritten in the form

$$
d=-j_{n} \cdot \nabla\left[\zeta_{n} / T\right]-j_{p} \cdot \nabla\left[\zeta_{p} / T\right]-j_{e} \cdot \nabla[-1 / T]+R\left(\zeta_{n}+\zeta_{p}\right) / T .
$$

Based on the foregoing discussion we introduce new variables $z=\left(z_{1}, z_{2}, z_{3}, z_{4}\right)=$ $\left(\zeta_{n} / T, \zeta_{p} / T,-1 / T, \varphi\right)$. The state equations (1) and the net recombination rate $R$ have to be expressed in terms of these variables,

$$
\begin{aligned}
& n(x)=N(x, T) F\left(\frac{\zeta_{n}+\varphi-E_{n}(x, T)}{T}\right)=H_{n}(x, z), \\
& p(x)=P(x, T) F\left(\frac{\zeta_{p}-\varphi+E_{p}(x, T)}{T}\right)=H_{p}(x, z),
\end{aligned}
$$




$$
R=r(x, n, p, T)\left(\mathrm{e}^{\left(\zeta_{n}+\zeta_{p}\right) / T}-1\right)=\widetilde{r}(x, z)\left(\mathrm{e}^{z_{1}+z_{2}}-1\right)=R(x, z) .
$$

Now the stationary energy model consisting of the equations (3), (4), (2) and complemented by the flux relations (12) can be written in the more compact form

$$
-\nabla \cdot\left(\begin{array}{llll}
a_{11} & a_{12} & a_{13} & 0 \\
a_{21} & a_{22} & a_{23} & 0 \\
a_{31} & a_{32} & a_{33} & 0 \\
0 & 0 & 0 & \varepsilon
\end{array}\right)\left(\begin{array}{l}
\nabla z_{1} \\
\nabla z_{2} \\
\nabla z_{3} \\
\nabla z_{4}
\end{array}\right)=\left(\begin{array}{l}
-R \\
-R \\
0 \\
f-H_{n}+H_{p}
\end{array}\right)
$$

where the coefficients $a_{i k}, i, k=1, \ldots, 3$, have to be considered as functions of $x$ and $z$ just like the quantities $R, H_{n}$ and $H_{p}$ while $\varepsilon$ and $f$ depend only on $x$. Since we assumed that the Dirichlet parts and the Neumann parts of the boundary coincide for all equations, we can write the boundary conditions (6) also in terms of $z$ and $\nabla z$,

$$
\begin{gathered}
z_{i}=z_{i}^{D}, \quad i=1, \ldots, 4, \quad \text { on } \Gamma_{D}, \\
\nu \cdot \sum_{k=1,2,3} a_{i k}(\cdot, z) \nabla z_{k}=g_{i}, \quad i=1, \ldots, 3, \quad \nu \cdot\left(\varepsilon \nabla z_{4}\right)=g_{4} \quad \text { on } \Gamma_{N} .
\end{gathered}
$$

1.2. Stationary energy model with multiple species. Next, we consider a more general stationary energy model with multiple species which applies to problems in electrochemistry. But in some applications (e.g. in semiconductor technology modelling) the stationary case for its own is of less interest. Nevertheless the study of the stationary model becomes important if one is interested in the long-time behaviour of solutions of the corresponding non-stationary model.

We are looking at a finite number of different species $X_{i}, i=1, \ldots, n$ (e.g. electrons, holes, dopants, interstitials, vacancies, dopant-defect pairs, clusters, etc. in semiconductor technology modelling). Let again $\varphi$ and $T$ be the electrostatic potential and the temperature, respectively. We denote by $u_{i}, \zeta_{i}, q_{i}$ the particle density of the species $X_{i}$, its electrochemical potential and its charge number. We use the state equations (based on the Boltzmann statistics, for example)

$$
u_{i}(x)=\bar{u}_{i}(x, T) \mathrm{e}^{\left(\zeta_{i}-q_{i} \varphi+E_{i}(x, T)\right) / T}, \quad i=1, \ldots, n,
$$

where $\bar{u}_{i}>0, E_{i}$ are suitable chosen reference quantities. The electrostatic potential fulfils the Poisson equation

$$
-\nabla \cdot(\varepsilon \nabla \varphi)=f+\sum_{i=1}^{n} q_{i} u_{i}
$$

Next, we consider a finite number of reversible reactions of the form

$$
\alpha_{1} X_{1}+\cdots+\alpha_{n} X_{n} \rightleftharpoons \beta_{1} X_{1}+\cdots+\beta_{n} X_{n}, \quad(\alpha, \beta) \in \mathcal{R}
$$

where $\alpha_{i}, \beta_{i} \in \mathbb{Z}_{+}$are the stoichiometric coefficients, and $\mathcal{R}$ denotes the set of pairs $(\alpha, \beta)=\left(\left(\alpha_{1}, \ldots, \alpha_{n}\right),\left(\beta_{1}, \ldots, \beta_{n}\right)\right)$ belonging to all reactions. According to the mass action law the reaction rates $R_{\alpha \beta}$ are given by

$$
R_{\alpha \beta}=r_{\alpha \beta}\left(\mathrm{e}^{\sum_{i=1}^{n} \alpha_{i} \zeta_{i} / T}-\mathrm{e}^{\sum_{i=1}^{n} \beta_{i} \zeta_{i} / T}\right), \quad(\alpha, \beta) \in \mathcal{R}
$$


where $r_{\alpha \beta}>0$ depends on $x$, on $u=\left(u_{1}, \ldots, u_{n}\right), T$, and on $\varphi$, maybe. We assume that each reaction preserves the charge, in other words that $\sum_{i}^{n}\left(\alpha_{i}-\beta_{i}\right) q_{i}=0$ for all $(\alpha, \beta) \in \mathcal{R}$. For the particle flux densities $j_{i}$ and the total energy flux density $j_{e}$ we make an ansatz similar to (5),

$$
\begin{aligned}
& j_{i}=-\sum_{k=1}^{n} \sigma_{i k}(x, u, T)\left(\nabla \zeta_{k}+P_{k}(x, u, T) \nabla T\right), \quad i=1, \ldots, n, \\
& j_{e}=-\kappa(x, u, T) \nabla T+\sum_{i=1}^{n}\left(\zeta_{i}+P_{i}(x, u, T) T\right) j_{i}
\end{aligned}
$$

with conductivities $\sigma_{i k}, \kappa$ fulfilling the relations

$$
\sigma_{i k}=\sigma_{k i}, \sum_{i, k=1}^{n} \sigma_{i k}(x, u, T) \xi_{i} \xi_{k} \geq \sigma_{0}(u, T) \sum_{i=1}^{n} \xi_{i}^{2} \quad \forall \xi \in \mathbb{R}^{n}, \kappa(x, u, T) \geq \kappa_{0}(u, T)
$$

where $\sigma_{0}(u, T), \kappa_{0}(u, T)>0$ for all non-degenerate states $0<u_{i}, T<\infty$. For the transported entropies $P_{i}$ we need no sign conditions.

Generalizing (3), (4) we have to pose $n$ continuity equations for all considered species and the conservation law for the total energy,

$$
\begin{aligned}
& \nabla \cdot j_{i}=R_{i}, \quad i=1, \ldots, n, \\
& \nabla \cdot j_{e}=0
\end{aligned}
$$

where the right hand sides of the continuity equations are given by

$$
R_{i}=\sum_{(\alpha, \beta) \in \mathcal{R}}\left(\beta_{i}-\alpha_{i}\right) R_{\alpha \beta}, \quad i=1, \ldots, n .
$$

The corresponding generalization of the boundary conditions (6) is obvious.

The further discussion follows the ideas in Subsection 1.1. We introduce the variables $z=\left(z_{1}, \ldots, z_{n+2}\right)=\left(\zeta_{1} / T, \ldots, \zeta_{n} / T,-1 / T, \varphi\right)$. The state equations $(15)$ and the reaction rates (17) are written in the form

$$
u_{i}=H_{i}(x, z), \quad i=1, \ldots, n, \quad R_{\alpha \beta}=\widetilde{r}_{\alpha \beta}(x, z)\left(\mathrm{e}^{\sum_{i=1}^{n} \alpha_{i} z_{i}}-\mathrm{e}^{\sum_{i=1}^{n} \beta_{i} z_{i}}\right), \quad(\alpha, \beta) \in \mathcal{R},
$$

and the differential equations $(20),(16)$ together with the flux relations (18) lead to

$$
-\nabla \cdot\left(\begin{array}{cccc}
a_{11} & \cdots & a_{1, n+1} & 0 \\
\vdots & \ddots & \vdots & 0 \\
a_{n, 1} & \cdots & a_{n, n+1} & 0 \\
a_{n+1,1} & \cdots & a_{n+1, n+1} & 0 \\
0 & 0 & 0 & \varepsilon
\end{array}\right)\left(\begin{array}{c}
\nabla z_{1} \\
\vdots \\
\nabla z_{n} \\
\nabla z_{n+1} \\
\nabla z_{n+2}
\end{array}\right)=\left(\begin{array}{c}
R_{1} \\
\vdots \\
R_{n} \\
0 \\
f-h_{0}
\end{array}\right)
$$

where $h_{0}$ is defined by $h_{0}(x, z)=-\sum_{i=1}^{n} q_{i} H_{i}(x, z)$ and the coefficients $a_{i k}$ are functions of $x$ and $z$. Finally, the boundary conditions are obtained as

$$
\begin{gathered}
z_{i}=z_{i}^{D}, i=1, \ldots, n+2, \quad \text { on } \Gamma_{D}, \\
\nu \cdot \sum_{k=1}^{n+1} a_{i k}(\cdot, z) \nabla z_{k}=g_{i}, i=1, \ldots, n+1, \quad \nu \cdot\left(\varepsilon \nabla z_{n+2}\right)=g_{n+2} \quad \text { on } \Gamma_{N} .
\end{gathered}
$$


The matrix $a_{i k}$ is symmetric and from (19) it follows that for each compact subset $K \subset \mathbb{R}^{n} \times(-\infty, 0) \times \mathbb{R}$ there exists a constant $a_{K}>0$ such that

$$
\sum_{i, k=1}^{n+1} a_{i k}(x, z) \xi_{i} \xi_{k} \geq a_{K} \sum_{i=1}^{n+1} \xi_{i}^{2}, \quad x \in \Omega, \quad z \in K, \quad \xi \in \mathbb{R}^{n+1} .
$$

Moreover, reasonable assumptions on the reference quantities in (15) ensure that for each compact subset $K \subset \mathbb{R}^{n} \times(-\infty, 0) \times \mathbb{R}$ there exists a constant $h_{K}>0$ such that

$$
\begin{gathered}
\left(h_{0}(x, z)-h_{0}(x, \bar{z})\right)\left(z_{n+2}-\bar{z}_{n+2}\right) \geq h_{K}\left|z_{n+2}-\bar{z}_{n+2}\right|^{2}, \\
x \in \Omega, \quad z, \bar{z} \in K \text { with } z_{i}=\bar{z}_{i} \text { for } i=1, \ldots, n+1 .
\end{gathered}
$$

Let us add some comments on thermodynamic equilibria. The entropy flux density $j_{s}=-\sum_{i=1}^{n+1} z_{i} j_{i}$ fulfils equation (11) where the production rate $d$ is given now by

$$
d=\sum_{i, k=1}^{n+1} a_{i k} \nabla z_{i} \cdot \nabla z_{k}+\sum_{(\alpha, \beta) \in \mathcal{R}} \widetilde{r}_{\alpha \beta}\left(\mathrm{e}^{\sum_{i=1}^{n} \alpha_{i} z_{i}}-\mathrm{e}^{\sum_{i=1}^{n} \beta_{i} z_{i}}\right) \sum_{i=1}^{n}\left(\alpha_{i}-\beta_{i}\right) z_{i} .
$$

For non-degenerate states we find that $d=0$ if and only if the equilibrium conditions $\nabla z_{i}=0, i=1, \ldots, n+1$, and $\sum_{i=1}^{n}\left(\alpha_{i}-\beta_{i}\right) z_{i}=0$ for all $(\alpha, \beta) \in \mathcal{R}$ are fulfilled. The necessary conditions on the data in the boundary conditions (22) are

$$
z_{i}^{D}=\text { const }, g_{i}=0, i=1, \ldots, n+1, \quad z_{n+1}^{D}<0, \quad \sum_{i=1}^{n}\left(\alpha_{i}-\beta_{i}\right) z_{i}^{D}=0 \forall(\alpha, \beta) \in \mathcal{R} .
$$

The corresponding electrostatic potential fulfils the nonlinear Poisson equation

$$
-\nabla \cdot\left(\varepsilon \nabla z_{n+2}\right)+h_{0}\left(z_{1}^{D}, \ldots, z_{n+1}^{D}, z_{n+2}\right)=f
$$

with mixed boundary conditions

$$
z_{n+2}=z_{n+2}^{D} \quad \text { on } \Gamma_{D}, \quad \nu \cdot\left(\varepsilon \nabla z_{n+2}\right)=g_{n+2} \quad \text { on } \Gamma_{N} .
$$

More precisely, the equilibria considered here are restricted equilibria (or Boltzmann equilibria) in an exterior field generated by the source terms $f, z_{n+2}^{D}, g_{n+2}$.

REmark 1. The model considered in Subsection 1.1 fits into the form (21), (22) also in the Fermi-Dirac case if our later assumptions on the function $h_{0}$ are formulated generally enough.

REMARK 2. The resulting problem is a boundary value problem which has non-smooth data in the following sense. Firstly, it is defined on a domain $\Omega$ which in general is nonsmooth, but only Lipschitz. Secondly, we have to deal with mixed boundary conditions where $\overline{\Gamma_{D}} \cap \overline{\Gamma_{N}} \neq \emptyset$. Thirdly, we want to consider also heterostructures and then the coefficients are discontinuous with respect to the space variable. Other difficulties arise from the following facts. The coefficients depend on the state variables. The system is strongly coupled and the ellipticity condition (23) is not fulfilled uniformly on $\Omega \times \mathbb{R}^{n} \times$ $(-\infty, 0] \times \mathbb{R}$. Finally, one has to take into account the constraint $z_{n+1}<0$.

1.3. Aim of the paper. The aim of the paper is to prove a local existence and uniqueness result near a thermodynamic equilibrium. For this purpose first we will ensure that for 
boundary data $z_{i}^{D}, g_{i}, i=1, \ldots, n+2$, which are compatible with thermodynamic equilibrium (see (25)) there exists a unique solution of $(21),(22)$. Then we use the Implicit Function Theorem to prove the existence of a unique solution of (21), (22) in a neighbourhood of this thermodynamic equilibrium. We can guarantee that $T>0, u_{i}>0, i=1, \ldots, n$, for these solutions. The main problem consists in finding a weak formulation of the stationary energy model in suitable function spaces such that the requirements of the Implicit Function Theorem can be verified. To obtain the necessary properties of differentiability we use properties of superposition operators established in [15]. Additionally, we take advantage of regularity results in [10] valid for strongly coupled elliptic systems with mixed boundary conditions and non-smooth data. Let us mention that the technique used here does not work in space dimensions greater than two.

2. Assumptions. Now we summarize all assumptions which we need in this paper. They are motivated by the examples considered in Section 1. We make use of

Definition 1 . Let $V=\mathbb{R}^{n} \times(-\infty, 0) \times \mathbb{R}$. We say that a function $b: \Omega \times V \rightarrow \mathbb{R}$ is of class (D) iff it fulfils the following properties:

$z \mapsto b(x, z)$ is continuously differentiable for almost all $x \in \Omega$,

$x \mapsto b(x, z)$ and $x \mapsto \partial_{z} b(x, z)$ are measurable for all $z \in V$,

for every compact subset $K \subset V$ there exists an $M>0$ such that

$|b(x, z)| \leq M$ and $\left\|\partial_{z} b(x, z)\right\| \leq M$ for all $z \in K$ and almost all $x \in \Omega$,

for every compact subset $K \subset V$ and $\epsilon>0$ there exists a $\delta>0$ such that

$|b(x, z)-b(x, \bar{z})|<\epsilon$ and $\left|\partial_{z} b(x, z)-\partial_{z} b(x, \bar{z})\right|<\epsilon$

for all $z, \bar{z} \in K$ with $|z-\bar{z}|<\delta$ and for almost all $x \in \Omega$.

Our assumptions are the following:

(A1) $\Omega$ is a bounded Lipschitzian domain in $\mathbb{R}^{2}, \Gamma=\partial \Omega$,

$\Gamma_{D}, \Gamma_{N}$ are disjoint open subsets of $\Gamma, \Gamma=\Gamma_{D} \cup \Gamma_{N} \cup\left(\overline{\Gamma_{D}} \cap \overline{\Gamma_{N}}\right), \operatorname{mes}_{D}>0$,

$\overline{\Gamma_{D}} \cap \overline{\Gamma_{N}}$ consists of finitely many points (see also Figure 1).

(A2) The functions $a_{i k}: \Omega \times V \rightarrow \mathbb{R}$ are of class (D), $i, k=1, \ldots, n+1$.

For every compact subset $K \subset V$ there exists an $a_{K}>0$ such that

$\sum_{i, k=1}^{n+1} a_{i k}(x, z) \xi_{i} \xi_{k} \geq a_{K}\|\xi\|^{2}$ for all $z \in K$, all $\xi \in \mathbb{R}^{n+1}$ and f.a.a. $x \in \Omega$.

(A3) $\varepsilon \in L^{\infty}(\Omega), 0<\varepsilon_{0} \leq \varepsilon(x) \leq \varepsilon^{0}<\infty$ almost everywhere in $\Omega$.

(A4) The function $h_{0}: \Omega \times V \rightarrow \mathbb{R}$ is of class (D),

$h_{0}\left(x, z_{1}, \ldots, z_{n+1}, \cdot\right)$ is monotonic increasing

for all $\left(z_{1}, \ldots, z_{n+1}\right) \in \mathbb{R}^{n} \times(-\infty, 0)$ and almost all $x \in \Omega$,

there are constants $c_{k}, c>0$ such that $\left|h_{0}\left(x, z_{1}, \ldots, z_{n+2}\right)\right| \leq c_{k} \mathrm{e}^{c\left|z_{n+2}\right|}$

for all $z \in[-k, k]^{n} \times[-k,-1 / k] \times \mathbb{R}$ and almost all $x \in \Omega$.

(A5) $\mathcal{R} \subset \mathbb{Z}_{+}^{n} \times \mathbb{Z}_{+}^{n}$, for $(\alpha, \beta)=\left(\left(\alpha_{1}, \ldots, \alpha_{n}\right),\left(\beta_{1}, \ldots, \beta_{n}\right)\right) \in \mathcal{R}$ we define

$R_{\alpha \beta}: \Omega \times V \rightarrow \mathbb{R}$ by $R_{\alpha \beta}(x, z)=\widetilde{r}_{\alpha \beta}(x, z)\left(\mathrm{e}^{\sum_{i=1}^{n} \alpha_{i} z_{i}}-\mathrm{e}^{\sum_{i=1}^{n} \beta_{i} z_{i}}\right)$

where $\tilde{r}_{\alpha \beta}: \Omega \times V \rightarrow \mathbb{R}_{+}$is of class (D). 
The data $z_{i}^{D}, g_{i}$ and $f$ in (21), (22) will be assumed to have at least the following properties. There exists a $p>2$ such that $z_{i}^{D}$ prescribed on $\Gamma_{D}$ is the trace of a function $z_{i}^{D} \in W^{1, p}(\Omega), i=1, \ldots, n+2$, with $z_{n+1}^{D}<0$ on $\Omega, g_{i} \in L^{\infty}\left(\Gamma_{N}\right), i=1, \ldots, n+2$, and $f \in L^{\infty}(\Omega)$.

3. Weak formulation of problem (21), (22). We define the vectors

$$
z^{D}=\left(z_{1}^{D}, \ldots, z_{n+2}^{D}\right), \quad g=\left(g_{1}, \ldots, g_{n+2}\right), \quad w=\left(z^{D}, g, f\right) .
$$

We are looking for solutions of $(21),(22)$ in the form

$$
z=Z+z^{D}
$$

where $z^{D}$ fulfils the Dirichlet boundary conditions of (22) and $Z$ represents the homogeneous part of the solution. We use the following function spaces:

$$
\begin{aligned}
& X_{s}=\left(W_{0}^{1, s}\left(\Omega \cup \Gamma_{N}\right)\right)^{n+2}, \quad Y_{s}=\left(W^{1, s}(\Omega)\right)^{n+2}, \\
& X_{s}^{*}=\left(\left(W_{0}^{1, s}\left(\Omega \cup \Gamma_{N}\right)\right)^{n+2}\right)^{*}=\left(W^{-1, s}\left(\Omega \cup \Gamma_{N}\right)\right)^{n+2}, \quad s \in[1, \infty) .
\end{aligned}
$$

Definition 2. Let $q \in(2, p]$ and $\tau>1$. We define the subset $M_{q, \tau} \subset X_{q} \times Y_{p}$ as

$$
\begin{aligned}
M_{q, \tau}=\left\{\left(Z, z^{D}\right) \in X_{q} \times Y_{p}:\left|Z_{i}+z_{i}^{D}\right|<\tau, i\right. & =1, \ldots, n, n+2 \\
& \left.-\tau<Z_{n+1}+z_{n+1}^{D}<-\frac{1}{\tau} \text { on } \Omega\right\} .
\end{aligned}
$$

Because of the continuous embedding $W^{1, p}, W^{1, q} \hookrightarrow C(\bar{\Omega})$ the set $M_{q, \tau}$ is open in $X_{q} \times Y_{p}$. Obviously, if $q_{2}>q_{1}$ then $M_{q_{2}, \tau} \subset M_{q_{1}, \tau}$, and if $\tau_{1}<\tau_{2}$ then $M_{q, \tau_{1}} \subset M_{q, \tau_{2}}$. We define the operator $F_{q, \tau}: M_{q, \tau} \times L^{\infty}\left(\Gamma_{N}\right)^{n+2} \times L^{\infty}(\Omega) \rightarrow X_{q^{\prime}}^{*}$ by

$$
\begin{aligned}
\left\langle F_{q, \tau}(Z, w), \psi\right\rangle_{X_{q^{\prime}}}= & \int_{\Omega}\left\{\sum_{i, k=1}^{n+1} a_{i k}(\cdot, z) \nabla z_{k} \cdot \nabla \psi_{i}+\varepsilon \nabla z_{n+2} \cdot \nabla \psi_{n+2}\right\} \mathrm{d} x \\
& +\int_{\Omega}\left\{\sum_{(\alpha, \beta) \in \mathcal{R}} R_{\alpha \beta}(\cdot, z) \sum_{i=1}^{n}\left(\alpha_{i}-\beta_{i}\right) \psi_{i}+h_{0}(\cdot, z) \psi_{n+2}\right\} \mathrm{d} x \\
& -\int_{\Omega} f \psi_{n+2} \mathrm{~d} x-\int_{\Gamma_{N}} \sum_{i=1}^{n+2} g_{i} \psi_{i} \mathrm{~d} \Gamma, \quad \psi \in X_{q^{\prime}} .
\end{aligned}
$$

Here $q^{\prime}=q /(q-1)$ denotes the dual exponent of $q$. The operator $F_{q, \tau}$ is defined on an open subset of $X_{q} \times Y_{p} \times L^{\infty}\left(\Gamma_{N}\right)^{n+2} \times L^{\infty}(\Omega)$. Using this notation a weak formulation of the system $(21),(22)$ is

Problem $(\mathrm{P})$. Find $(q, \tau, Z, w)$ such that $q \in(2, p], \tau>1,(Z, w) \in X_{q} \times Y_{p} \times$ $L^{\infty}\left(\Gamma_{N}\right)^{n+2} \times L^{\infty}(\Omega)$,

$$
F_{q, \tau}(Z, w)=0, \quad\left(Z, z^{D}\right) \in M_{q, \tau}
$$

If $(q, \tau, Z, w)$ is a solution of $(\mathrm{P})$ then $(\widetilde{q}, \widetilde{\tau}, Z, w)$ with $\widetilde{q} \in(2, q]$ and $\widetilde{\tau} \geq \tau$ is a solution of $(\mathrm{P})$, too. 


\section{Results}

LEMma 1 (Differentiability). Assume (A1)-(A5). The operator $F_{q, \tau}: M_{q, \tau} \times L^{\infty}\left(\Gamma_{N}\right)^{n+2}$ $\times L^{\infty}(\Omega) \rightarrow X_{q^{\prime}}^{*}$ is continuously differentiable for all exponents $q \in(2, p]$ and all parameters $\tau>1$.

Proof. Let $q \in(2, p]$ and $\tau>1$ be arbitrarily fixed. We split up the operator $F_{q, \tau}$ in the form $F_{q, \tau}=A^{0}+A^{1}-B$ where $A^{0}, A^{1}: M_{q, \tau} \rightarrow X_{q^{\prime}}^{*}, B: L^{\infty}\left(\Gamma_{N}\right)^{n+2} \times L^{\infty}(\Omega) \rightarrow X_{q^{\prime}}^{*}$,

$$
\begin{aligned}
\left\langle A^{0}\left(Z, z^{D}\right), \psi\right\rangle_{X_{q^{\prime}}} & =\int_{\Omega}\left\{\sum_{i, k=1}^{n+1} a_{i k}(\cdot, z) \nabla Z_{k} \cdot \nabla \psi_{i}+\varepsilon \nabla Z_{n+2} \cdot \nabla \psi_{n+2}\right\} \mathrm{d} x \\
& +\int_{\Omega}\left\{\sum_{(\alpha, \beta) \in \mathcal{R}} R_{\alpha \beta}(\cdot, z) \sum_{i=1}^{n}\left(\alpha_{i}-\beta_{i}\right) \psi_{i}+h_{0}(\cdot, z) \psi_{n+2}\right\} \mathrm{d} x, \\
\left\langle A^{1}\left(Z, z^{D}\right), \psi\right\rangle_{X_{q^{\prime}}} & =\int_{\Omega}\left\{\sum_{i, k=1}^{n+1} a_{i k}(\cdot, z) \nabla z_{k}^{D} \cdot \nabla \psi_{i}+\varepsilon \nabla z_{n+2}^{D} \cdot \nabla \psi_{n+2}\right\} \mathrm{d} x, \\
\langle B(g, f), \psi\rangle_{X_{q^{\prime}}} & =\int_{\Omega} f \psi_{n+2} \mathrm{~d} x+\int_{\Gamma_{N}} \sum_{i=1}^{n+2} g_{i} \psi_{i} \mathrm{~d} \Gamma, \quad \psi \in X_{q^{\prime}} .
\end{aligned}
$$

For the proof for the part $A^{0}: M_{q, \tau} \rightarrow X_{q^{\prime}}^{*}$ we refer to [15, p. 1465, Lemma 2.2]. Again using [15, Lemma 2.2] we find that $A^{1}: M_{q, \tau} \rightarrow X_{p^{\prime}}^{*}$ is continuously differentiable, and the continuous embedding $W^{1, p}(\Omega) \hookrightarrow W^{1, q}(\Omega)$ gives the result for $A^{1}: M_{q, \tau} \rightarrow X_{q^{*}}^{*}$. Note that our assumptions guarantee the validity of (H2.1), (H2.2), (H2.3) in [15]. Assertions concerning the operator $B$ are trivial. Especially we have

$$
\begin{aligned}
\left\langle\partial_{Z} F_{q, \tau}(Z, w) \bar{Z}, \psi\right\rangle_{X_{q^{\prime}}} & =\int_{\Omega} \sum_{i, k=1}^{n+1}\left(a_{i k}(\cdot, z) \nabla \bar{Z}_{k}+\partial_{z} a_{i k}(\cdot, z) \cdot \bar{Z} \nabla z_{k}\right) \cdot \nabla \psi_{i} \mathrm{~d} x \\
& +\int_{\Omega}\left\{\varepsilon \nabla \bar{Z}_{n+2} \cdot \nabla \psi_{n+2}+\partial_{z} h_{0}(\cdot, z) \cdot \bar{Z} \psi_{n+2}\right\} \mathrm{d} x \\
& +\int_{\Omega} \sum_{(\alpha, \beta) \in \mathcal{R}} \partial_{z} R_{\alpha \beta}(\cdot, z) \cdot \bar{Z} \sum_{i=1}^{n}\left(\alpha_{i}-\beta_{i}\right) \psi_{i} \mathrm{~d} x
\end{aligned}
$$

for all $\bar{Z} \in X_{q}$ and $\psi \in X_{q^{\prime}}$.

For $p>2$ fixed we define the set of data which are compatible with thermodynamic equilibria (see $(25))$

$$
\begin{gathered}
Q=\left\{w=\left(z^{D}, g, f\right) \in Y_{p} \times L^{\infty}\left(\Gamma_{N}\right)^{n+2} \times L^{\infty}(\Omega): z_{i}^{D}=\text { const, } g_{i}=0, i=1, \ldots, n+1,\right. \\
\left.\sum_{i=1}^{n}\left(\alpha_{i}-\beta_{i}\right) z_{i}^{D}=0 \quad \forall(\alpha, \beta) \in \mathcal{R}, \quad z_{n+1}^{D}<0\right\} .
\end{gathered}
$$

TheOREM 1 (Existence and uniqueness of thermodynamic equilibria). We make the assumptions (A1)-(A5). Let $w^{*}=\left(z^{D *}, g^{*}, f^{*}\right) \in Q$ be given .

i) Then there exist a $q_{0} \in(2, p]$, a constant $\tau>1$ and a function $Z_{n+2}^{*} \in W_{0}^{1, q_{0}}\left(\Omega \cup \Gamma_{N}\right)$ such that $\left(Z^{*}, z^{D *}\right)=\left(\left(0, \ldots, 0, Z_{n+2}^{*}\right), z^{D *}\right) \in M_{q_{0}, \tau}$ and $F_{q_{0}, \tau}\left(Z^{*}, w^{*}\right)=0$. In other words, $\left(q_{0}, \tau, Z^{*}, w^{*}\right)$ is a solution of $(\mathrm{P})$. 
ii) $z^{*}=Z^{*}+z^{D *}$ is a thermodynamic equilibrium of (21), (22).

iii) If $\left(\widetilde{q}, \widetilde{\tau}, \widetilde{Z}, w^{*}\right)$ is a solution of $(\mathrm{P})$, then $\widetilde{Z}=Z^{*}$ in $X_{\widehat{q}}$ with $\widehat{q}=\min \left\{q_{0}, \widetilde{q}\right\}$.

Proof. 1. For the given $w^{*}=\left(z^{D *}, g^{*}, f^{*}\right)$ we define the function $h_{1}: \Omega \times \mathbb{R} \rightarrow \mathbb{R}$ by

$$
h_{1}(x, \phi)=h_{0}\left(x,(0, \ldots, 0, \phi)+z^{D *}\right)
$$

and consider the operator $\mathcal{E}: H_{0}^{1}\left(\Omega \cup \Gamma_{N}\right) \rightarrow H^{-1}\left(\Omega \cup \Gamma_{N}\right)$,

$$
\begin{aligned}
\langle\mathcal{E}(\phi), \bar{\phi}\rangle_{H_{0}^{1}\left(\Omega \cup \Gamma_{N}\right)} & =\int_{\Omega} \varepsilon \nabla\left(\phi+z_{n+2}^{D *}\right) \cdot \nabla \bar{\phi} \mathrm{d} x-\int_{\Gamma_{N}} g_{n+2}^{*} \bar{\phi} \mathrm{d} \Gamma \\
& +\int_{\Omega}\left(h_{1}(\cdot, \phi)-f^{*}\right) \bar{\phi} \mathrm{d} x \quad \forall \bar{\phi} \in H_{0}^{1}\left(\Omega \cup \Gamma_{N}\right) .
\end{aligned}
$$

For $\phi_{1}, \phi_{2} \in H_{0}^{1}\left(\Omega \cup \Gamma_{N}\right)$ we have

$$
\begin{aligned}
& \left\langle\mathcal{E}\left(\phi_{1}\right)-\mathcal{E}\left(\phi_{2}\right), \phi_{1}-\phi_{2}\right\rangle_{H_{0}^{1}\left(\Omega \cup \Gamma_{N}\right)} \\
& =\int_{\Omega}\left\{\varepsilon\left|\nabla\left(\phi_{1}-\phi_{2}\right)\right|^{2}+\left(h_{1}\left(\cdot, \phi_{1}\right)-h_{1}\left(\cdot, \phi_{2}\right)\right)\left(\phi_{1}-\phi_{2}\right)\right\} \mathrm{d} x,
\end{aligned}
$$

and the properties (A1),(A3),(A4) of $\Gamma_{D}, \varepsilon$ and $h_{0}$ yield the strong monotonicity of the operator $\mathcal{E}$. Next we prove the hemicontinuity of $\mathcal{E}$. We have to show that the mapping $t \mapsto\langle\mathcal{E}(\phi+t \widehat{\phi}), \bar{\phi}\rangle_{H_{0}^{1}\left(\Omega \cup \Gamma_{N}\right)}$ for fixed $\phi, \widehat{\phi}, \bar{\phi} \in H_{0}^{1}\left(\Omega \cup \Gamma_{N}\right)$ is continuous on [0,1]. Let $t_{0} \in[0,1], t_{n} \rightarrow t_{0}, t_{n} \in[0,1]$. Then

$$
\begin{aligned}
& \left\langle\mathcal{E}\left(\phi+t_{n} \widehat{\phi}\right)-\mathcal{E}\left(\phi+t_{0} \widehat{\phi}\right), \bar{\phi}\right\rangle_{H_{0}^{1}\left(\Omega \cup \Gamma_{N}\right)} \\
& \leq c\left|t_{n}-t_{0}\right|\|\widehat{\phi}\|_{H^{1}}\|\bar{\phi}\|_{H^{1}}+\left|\int_{\Omega}\left[h_{1}\left(\cdot, \phi+t_{n} \widehat{\phi}\right)-h_{1}\left(\cdot, \phi+t_{0} \widehat{\phi}\right)\right] \bar{\phi} \mathrm{d} x\right| .
\end{aligned}
$$

According to (A4) we have $h_{1}\left(x, \phi+t_{n} \widehat{\phi}\right) \rightarrow h_{1}\left(x, \phi+t_{0} \widehat{\phi}\right)$ and

$$
\left|h_{1}\left(x, \phi+t_{n} \widehat{\phi}\right)\right| \leq \widetilde{c} \mathrm{e}^{\widetilde{c}(|\phi|+|\widehat{\phi}|)} \text { for almost all } x \in \Omega .
$$

Now we use the embedding result of Trudinger [17] for two dimensional Lipschitzian domains which tells us that

$$
\left\|\mathrm{e}^{|v|}\right\|_{L^{2}} \leq d\left(\|v\|_{H^{1}}\right) \quad \forall v \in H^{1}(\Omega)
$$

where $d: \mathbb{R}_{+} \rightarrow \mathbb{R}_{+}$is a continuous, monotonic increasing function, $\lim _{y \rightarrow \infty} d(y)=\infty$. Since $\bar{\phi} \in L^{2}(\Omega)$ we get an integrable upper bound for the integrand in the last term in (28) and Lebesgue's Dominated Convergence Theorem leads to the hemicontinuity of $\mathcal{E}$. Since $\mathcal{E}$ is strongly monotone and hemicontinuous there exists a unique solution $\phi \in H_{0}^{1}\left(\Omega \cup \Gamma_{N}\right)$ of $\mathcal{E}(\phi)=0$, and $\|\phi\|_{H^{1}} \leq \widehat{c}$ where $\widehat{c}$ depends only on the data $w^{*}$.

2. Next we prove that this solution possesses more regularity. We define

$$
\begin{aligned}
\left\langle\mathcal{E}_{0}(\phi), \bar{\phi}\right\rangle_{H_{0}^{1}\left(\Omega \cup \Gamma_{N}\right)} & =\int_{\Omega}\{\varepsilon \nabla \phi \cdot \nabla \bar{\phi}+\phi \bar{\phi}\} \mathrm{d} x \\
\langle\mathcal{T}, \bar{\phi}\rangle_{H_{0}^{1}\left(\Omega \cup \Gamma_{N}\right)} & =\int_{\Omega}\left\{-\varepsilon \nabla z_{n+2}^{D *} \cdot \nabla \bar{\phi}+\left(f^{*}-h_{1}(\cdot, \phi)+\phi\right) \bar{\phi}\right\} \mathrm{d} x \\
& +\int_{\Gamma_{N}} g_{n+2}^{*} \bar{\phi} \mathrm{d} \Gamma \quad \forall \bar{\phi} \in H_{0}^{1}\left(\Omega \cup \Gamma_{N}\right) .
\end{aligned}
$$


Since $z_{n+2}^{D *} \in W^{1, p}(\Omega)$ is a fixed element there is a $\bar{c}>0$ such that $\left|z_{n+2}^{D *}\right| \leq \bar{c}$. From the properties (A4) of $h_{0}$ we obtain $\left|h_{1}(x, \phi)\right| \leq c\left(z^{D *}\right) \mathrm{e}^{c\left|z_{n+2}^{D *}+\phi\right|} \leq \widetilde{c}\left(z^{D *}\right) \mathrm{e}^{c \bar{c}|\phi|}$ for almost all $x \in \Omega$. And therefore the embedding result of Trudinger mentioned in the first step of this proof yields

$$
\left\|h_{1}(\cdot, \phi)\right\|_{L^{2}} \leq \widetilde{c}\left(z^{D *}\right) d\left(\|\phi\|_{H^{1}}\right) \leq \widehat{c} .
$$

Furthermore, using that $z_{n+2}^{D *} \in W^{1, p}(\Omega),\left(g^{*}, f^{*}\right) \in L^{\infty}\left(\Gamma_{N}\right)^{n+2} \times L^{\infty}(\Omega)$ are fixed it results that $\mathcal{T} \in W^{-1, p}\left(\Omega \cup \Gamma_{N}\right)$. Thus from Gröger's regularity result [10] applied to the equation $\mathcal{E}_{0}(\phi)=\mathcal{T}$ we find a $q_{0} \in(2, p]$ such that $\phi \in W^{1, q_{0}}\left(\Omega \cup \Gamma_{N}\right)$ and $\|\phi\|_{W^{1, q_{0}}} \leq c_{q_{0}}\|\mathcal{T}\|_{W^{-1, p}\left(\Omega \cup \Gamma_{N}\right)}$. Note that our assumptions (A1) concerning the domain $\Omega$ and its boundary ensure that $\Omega \cup \Gamma_{N}$ is regular in the sense of Gröger.

3. The continuous embedding $W^{1, q_{0}}(\Omega) \hookrightarrow C(\bar{\Omega})$ ensures that $\left\|\phi+z_{n+2}^{D *}\right\|_{C(\bar{\Omega})} \leq$ $c\left(q_{0}, w^{*}\right)$. Setting $Z_{i}^{*}=0, i=1, \ldots, n+1, Z_{n+2}^{*}=\phi$ and using that $w^{*} \in Q$ we find a constant $\tau>1$ such that $\left(Z^{*}, z^{D *}\right) \in M_{q_{0}, \tau}$ and $F_{q_{0}, \tau}\left(Z^{*}, w^{*}\right)=0$. In other words, $\left(q_{0}, \tau, Z^{*}, w^{*}\right)$ is a solution of Problem $(\mathrm{P})$. Moreover, $z^{*}=Z^{*}+z^{D *}$ is a thermodynamic equilibrium of (21), (22).

4. Let $\left(\widetilde{q}, \widetilde{\tau}, \widetilde{Z}, w^{*}\right)$ be a solution of Problem $(\mathrm{P})$ and set $\widetilde{z}=\widetilde{Z}+z^{D *}$. Then we have $\left(Z^{*}, z^{D *}\right) \in M_{q_{0}, \tau},\left(\widetilde{Z}, z^{D *}\right) \in M_{\widetilde{q}, \widetilde{\tau}}$ and $F_{q_{0}, \tau}\left(Z^{*}, w^{*}\right)=F_{\widetilde{q}, \widetilde{\tau}}\left(\widetilde{Z}, w^{*}\right)=0$. We define $\widehat{q}=\min \left\{q_{0}, \widetilde{q}\right\}, \widehat{\tau}=\max \{\tau, \widetilde{\tau}\}$ and find that $\left(Z^{*}, z^{D *}\right),\left(\widetilde{Z}, z^{D *}\right) \in M_{\widehat{q}, \widehat{\tau}}, F_{\widehat{q}, \widehat{\tau}}\left(Z^{*}, w^{*}\right)=$ $F_{\widehat{q}, \widehat{\tau}}\left(\widetilde{Z}, w^{*}\right)=0$ and $F_{\widehat{q}, \widehat{\tau}}\left(\widetilde{Z}, w^{*}\right)-F_{\widehat{q}, \widehat{\tau}}\left(Z^{*}, w^{*}\right)=0$. We test the last equation with $\left(\widetilde{Z}_{1}, \ldots, \widetilde{Z}_{n+1}, 0\right)$. Since $w^{*}, w^{*}+\left(Z^{*}, 0,0\right) \in Q$ we obtain

$$
\begin{aligned}
0 & =\left\langle F_{\widehat{q}, \widehat{\tau}}\left(\widetilde{Z}, w^{*}\right)-F_{\widehat{q}, \widehat{\tau}}\left(Z^{*}, w^{*}\right),\left(\widetilde{Z}_{1}, \ldots, \widetilde{Z}_{n+1}, 0\right)\right\rangle_{W_{0}^{1, \widehat{q}^{\prime}}\left(\Omega \cup \Gamma_{N}\right)} \\
& =\int_{\Omega} \sum_{i, k=1}^{n+1} a_{i k}(\cdot, \widetilde{z}) \nabla \widetilde{Z}_{k} \cdot \nabla \widetilde{Z}_{i} \mathrm{~d} x \\
& +\int_{\Omega} \sum_{(\alpha, \beta) \in \mathcal{R}} \widetilde{r}_{\alpha \beta}(\cdot, \widetilde{z}) \mathrm{e}^{\sum_{i=1}^{n} \alpha_{i} z_{i}^{D *}}\left(\mathrm{e}^{\sum_{i=1}^{n} \alpha_{i} \widetilde{Z}_{i}}-\mathrm{e}^{\sum_{i=1}^{n} \beta_{i} \widetilde{Z}_{i}}\right) \sum_{i=1}^{n}\left(\alpha_{i}-\beta_{i}\right) \widetilde{Z}_{i} \mathrm{~d} x .
\end{aligned}
$$

Exploiting assumption (A5) for $\widetilde{r}_{\alpha \beta}$ and the fact that $\left(\mathrm{e}^{x}-\mathrm{e}^{y}\right)(x-y) \geq 0$ we find

$$
\int_{\Omega} \sum_{i, k=1}^{n+1} a_{i k}(\cdot, \widetilde{z}) \nabla \widetilde{Z}_{k} \cdot \nabla \widetilde{Z}_{i} \mathrm{~d} x \leq 0 .
$$

Since according to (A2) the matrix $\left(a_{i k}(x, \widetilde{z})\right)_{i, k=1, \ldots, n+1}$ is strongly elliptic we obtain $\nabla \widetilde{Z}_{i}=0$ and $\Gamma_{D} \neq \emptyset$ implies that $\widetilde{Z}_{i}=0, i=1, \ldots, n+1$. Finally, the test of the equation $F_{\widehat{q}, \widehat{\tau}}\left(\widetilde{Z}, w^{*}\right)-F_{\widehat{q}, \widehat{\tau}}\left(Z^{*}, w^{*}\right)=0$ with $\left(0, \ldots, 0, \widetilde{Z}_{n+2}-Z_{n+2}^{*}\right)$ leads to $\widetilde{Z}_{n+2}=Z_{n+2}^{*}$ since $\mathcal{E}$ is strongly monotone. In summary we obtain $\widetilde{Z}=Z^{*}$ which gives assertion iii).

LEMma 2 (Fredholm property of the linearization). We assume (A1)-(A5). Let $w^{*}=$ $\left(z^{D *}, g^{*}, f^{*}\right) \in Q$ be given. Let $\left(q_{0}, \tau, Z^{*}, w^{*}\right)$ be the equilibrium solution of Problem $(\mathrm{P})$ and $z^{*}=Z^{*}+z^{D *}$. Then there exists a $q_{1} \in\left(2, q_{0}\right]$ such that the operator $\partial_{Z} F_{q_{1}, \tau}\left(Z^{*}, w^{*}\right)$ is a Fredholm operator of index zero.

Proof. Let $q \in\left(2, q_{0}\right]$. The linearization is given in $(27)$ and has to be evaluated now at the point $\left(Z^{*}, w^{*}\right)$. Since $\nabla z_{i}^{*}=0, i=1, \ldots, n+1, \sum_{i=1}^{n} \alpha_{i} z_{i}^{*}=\sum_{i=1}^{n} \beta_{i} z_{i}^{*}$ and 


$$
\begin{aligned}
\partial_{z} R_{\alpha \beta}\left(\cdot, z^{*}\right) \cdot \bar{Z} & =\partial_{z} \widetilde{r}_{\alpha \beta}\left(\cdot, z^{*}\right) \cdot \bar{Z}\left(\mathrm{e}^{\sum_{i=1}^{n} \alpha_{i} z_{i}^{*}}-\mathrm{e}^{\sum_{i=1}^{n} \beta_{i} z_{i}^{*}}\right) \\
& +\widetilde{r}_{\alpha \beta}\left(\cdot, z^{*}\right) \sum_{k=1}^{n}\left(\alpha_{k} \mathrm{e}^{\sum_{i=1}^{n} \alpha_{i} z_{i}^{*}}-\beta_{k} \mathrm{e}^{\sum_{i=1}^{n} \beta_{i} z_{i}^{*}}\right) \bar{Z}_{k}
\end{aligned}
$$

we obtain according to $(27)$ that

$$
\begin{aligned}
& \left\langle\partial_{Z} F_{q, \tau}\left(Z^{*}, w^{*}\right) \bar{Z}, \psi\right\rangle_{X_{q^{\prime}}} \\
& \quad=\int_{\Omega}\left\{\sum_{i, k=1}^{n+1} a_{i k}\left(\cdot, z^{*}\right) \nabla \bar{Z}_{k} \cdot \nabla \psi_{i}+\varepsilon \nabla \bar{Z}_{n+2} \cdot \nabla \psi_{n+2}\right\} \mathrm{d} x \\
& \quad+\int_{\Omega} \sum_{(\alpha, \beta) \in \mathcal{R}} \tilde{r}_{\alpha \beta}\left(\cdot, z^{*}\right) \mathrm{e}^{\sum_{l=1}^{n} \alpha_{l} z_{l}^{*}} \sum_{k=1}^{n}\left(\alpha_{k}-\beta_{k}\right) \bar{Z}_{k} \sum_{i=1}^{n}\left(\alpha_{i}-\beta_{i}\right) \psi_{i} \mathrm{~d} x \\
& \quad+\int_{\Omega} \partial_{z} h_{0}\left(\cdot, z^{*}\right) \cdot \bar{Z} \psi_{n+2} \mathrm{~d} x, \quad \bar{Z} \in X_{q}, \quad \psi \in X_{q^{\prime}} .
\end{aligned}
$$

Now we follow ideas in the proof of [15, Theorem 4.1]. We write $\partial_{Z} F_{q, \tau}\left(Z^{*}, w^{*}\right)$ in the form $\partial_{Z} F_{q, \tau}\left(Z^{*}, w^{*}\right)=L_{q}+K_{q}$ where the operators $L_{q}, K_{q}: X_{q} \rightarrow X_{q^{\prime}}^{*}$ are defined by

$$
\begin{aligned}
\left\langle L_{q} \bar{Z}, \psi\right\rangle_{X_{q^{\prime}}} & =\int_{\Omega}\left\{\sum_{i, k=1}^{n+1} a_{i k}\left(\cdot, z^{*}\right) \nabla \bar{Z}_{k} \cdot \nabla \psi_{i}+\varepsilon \nabla \bar{Z}_{n+2} \cdot \nabla \psi_{n+2}+\sum_{i=1}^{n+2} \bar{Z}_{i} \psi_{i}\right\} \mathrm{d} x, \\
\left\langle K_{q} \bar{Z}, \psi\right\rangle_{X_{q^{\prime}}} & =\int_{\Omega} \sum_{(\alpha, \beta) \in \mathcal{R}} \widetilde{r}_{\alpha \beta}\left(\cdot, z^{*}\right) \mathrm{e}^{\sum_{l=1}^{n} \alpha_{l} z_{l}^{*}} \sum_{k=1}^{n}\left(\alpha_{k}-\beta_{k}\right) \bar{Z}_{k} \sum_{i=1}^{n}\left(\alpha_{i}-\beta_{i}\right) \psi_{i} \mathrm{~d} x \\
& +\int_{\Omega}\left\{\partial_{z} h_{0}\left(\cdot, z^{*}\right) \cdot \bar{Z} \psi_{n+2}-\sum_{i=1}^{n+2} \bar{Z}_{i} \psi_{i}\right\} \mathrm{d} x, \quad \bar{Z} \in X_{q}, \quad \psi \in X_{q^{\prime}} .
\end{aligned}
$$

The operator $K_{q}$ is compact because of the compact embedding $W^{1, q}(\Omega) \hookrightarrow L^{\infty}(\Omega)$. The operator $L_{q}$ is injective. The regularity result of Gröger [10, Theorem 1, Remark 14] guarantees that there exists a $q_{1} \in\left(2, q_{0}\right]$ such that $L_{q_{1}}$ is surjective. Then by Banach's Open Mapping Theorem and Nikolsky's criterion for Fredholm operators the assertion follows.

Lemma 3 (Injectivity of the linearization). We assume (A1)-(A5). Let $w^{*}=\left(z^{D *}, g^{*}, f^{*}\right)$ $\in Q$ be given. Let $\left(q_{0}, \tau, Z^{*}, w^{*}\right)$ be the equilibrium solution of Problem $(\mathrm{P})$ and $z^{*}=$ $Z^{*}+z^{D *}$. Then the linearization $\partial_{Z} F_{q_{1}, \tau}\left(Z^{*}, w^{*}\right): X_{q_{1}} \rightarrow X_{q_{1}^{\prime}}^{*}$ is injective where $q_{1}$ is chosen as in Lemma 2.

Proof. It is sufficient to prove the injectivity of the operator on $X_{2}$. The derivative $\partial_{Z} F_{q_{1}, \tau}\left(Z^{*}, w^{*}\right)$ has the form (29). Let $\partial_{Z} F_{q_{1}, \tau}\left(Z^{*}, w^{*}\right) \bar{Z}=0, \bar{Z} \in X_{2}$. We test this equation with $\psi=\left(\bar{Z}_{1}, \ldots, \bar{Z}_{n+1}, 0\right)$ and take into account the strong ellipticity condition for $\left(a_{i k}\left(x, z^{*}\right)\right)_{i, k=1, \ldots, n+1}$, the fact that $\Gamma_{D} \neq \emptyset$ and the property that $\widetilde{r}_{\alpha \beta}\left(\cdot, z^{*}\right) \mathrm{e}^{\sum_{k=1}^{n} \alpha_{k} z_{k}^{*}} \geq$ 0 for all $(\alpha, \beta) \in \mathcal{R}$ and find that $\bar{Z}_{i}=0, i=1, \ldots, n+1$. Next we use the test function $\psi=\left(0, \ldots, 0, \bar{Z}_{n+2}\right)$ and get

$$
\int_{\Omega}\left\{\varepsilon\left|\nabla \bar{Z}_{n+2}\right|^{2}+\frac{\partial}{\partial z_{n+2}} h_{0}\left(\cdot, z^{*}\right) \bar{Z}_{n+2}^{2}\right\} \mathrm{d} x=0 .
$$

Since $h_{0}$ is continuously differentiable and monotonic increasing in the argument $z_{n+2}$ 
(see $(\mathrm{A} 4))$ we have $\frac{\partial}{\partial z_{n+2}} h_{0}\left(x, z^{*}\right) \geq 0$ a.e. on $\Omega$ which together with $\varepsilon \geq \varepsilon_{0}$ a.e. on $\Omega$ leads to $\bar{Z}_{n+2}=0$. Thus also the injectivity of $\partial_{Z} F_{q_{1}, \tau}\left(Z^{*}, w^{*}\right): X_{q_{1}} \rightarrow X_{q_{1}^{\prime}}^{*}$ follows.

Now we are able to formulate and prove the main result of the paper.

THEOREM 2 (Local existence and uniqueness of steady states). We assume (A1)-(A5). Let $w^{*}=\left(z^{D *}, g^{*}, f^{*}\right) \in Q$ be given, and let $\left(q_{0}, \tau, Z^{*}, w^{*}\right)$ be the equilibrium solution of Problem $(\mathrm{P}), z^{*}=Z^{*}+z^{D *}=\left(0, \ldots, 0, Z_{n+2}^{*}\right)+z^{D *}$ (see Theorem 1$)$.

Then there exists a $q_{1} \in\left(2, q_{0}\right]$ such that the following assertion holds: There exist neighbourhoods $U \subset X_{q_{1}}$ of $Z^{*}$ and $W \subset Y_{p} \times L^{\infty}\left(\Gamma_{N}\right)^{n+2} \times L^{\infty}(\Omega)$ of $w^{*}=\left(z^{D *}, g^{*}, f^{*}\right)$ and a $C^{1}-$ map $\Phi: W \rightarrow U$ such that $Z=\Phi(w)$ iff

$$
F_{q_{1}, \tau}(Z, w)=0, \quad\left(Z, z^{D}\right) \in M_{q_{1}, \tau}, \quad Z \in U, \quad w=\left(z^{D}, g, f\right) \in W .
$$

Proof. According to Lemma 2 and Lemma 3 there is a $q_{1}$ such that the operator $\partial_{Z} F_{q_{1}, \tau}\left(Z^{*}, w^{*}\right): X_{q_{1}} \rightarrow X_{q_{1}^{\prime}}^{*}$ is an injective Fredholm operator of index zero. Therefore the assertion of the theorem is a consequence of the Implicit Function Theorem.

Finally, let us draw two conclusions from Theorem 2. Firstly, we define the set

$$
\begin{gathered}
Q_{1}=\left\{w=\left(z^{D}, g, f\right) \in Y_{p} \times L^{\infty}\left(\Gamma_{N}\right)^{n+2} \times L^{\infty}(\Omega): g_{i}=0, i=1, \ldots, n+1,\right. \\
\left.\int_{\Gamma_{D}} \sum_{i=1}^{n}\left(\alpha_{i}-\beta_{i}\right) z_{i}^{D} \mathrm{~d} \Gamma=0 \quad \forall(\alpha, \beta) \in \mathcal{R}, \quad z_{n+1}^{D}<0\right\} .
\end{gathered}
$$

Obviously $Q \subset Q_{1}$, but $Q_{1}$ contains also elements which are not compatible with thermodynamic equilibria.

Corollary 1. We assume (A1)-(A5). Let $w=\left(z^{D}, g, f\right) \in Q_{1}$ be given. Then there are constants $q \in(2, p], \tau>1, \epsilon>0$ such that the following assertions hold: If

$$
\left\|\nabla z_{i}^{D}\right\|_{L^{p}(\Omega)}<\epsilon, \quad i=1, \ldots, n+1,
$$

then there exists a $Z \in X_{q}$ such that $(q, \tau, Z, w)$ is a solution of $(\mathrm{P})$. This solution lies in a neighbourhood of an equilibrium solution $\left(q, \tau, Z^{*}, w^{*}\right)$ of $(\mathrm{P})$, and in this neighbourhood there are no solutions $(q, \tau, \widetilde{Z}, w)$ with $\widetilde{Z} \neq Z$.

Proof. Let $w=\left(z^{D}, g, f\right) \in Q_{1}$ be given. We define

$$
z_{i}^{D *}=\frac{1}{\left|\Gamma_{D}\right|} \int_{\Gamma_{D}} z_{i}^{D} \mathrm{~d} \Gamma, i=1, \ldots, n+1, \quad z_{n+2}^{D *}=z_{n+2}^{D}, \quad w^{*}=\left(z^{D *}, g, f\right)
$$

and find that $w^{*} \in Q$. Let $\left(q_{0}, \tau, Z^{*}, w^{*}\right)$ be the equilibrium solution of $(\mathrm{P})$. Because of Theorem 2 there exist constants $q \in\left(2, q_{0}\right], \epsilon^{\prime}>0$ such that the equation $F_{q, \tau}(Z, w)=0$ has a locally unique solution $Z \in X_{q}$ if

$$
\left\|w-w^{*}\right\|_{Y_{p} \times L^{\infty}\left(\Gamma_{N}\right)^{n+2} \times L^{\infty}(\Omega)}=\sum_{i=1}^{n+1}\left\|z_{i}^{D}-z_{i}^{D *}\right\|_{W^{1, p}(\Omega)}<\epsilon^{\prime} .
$$

Since for $i=1, \ldots, n+1$ the mean values of $z_{i}^{D}-z_{i}^{D *}$ on $\Gamma_{D}$ vanish we can apply the Friedrichs inequality to obtain

$$
\left\|z_{i}^{D}-z_{i}^{D *}\right\|_{W^{1, p}(\Omega)} \leq c\left\|\nabla z_{i}^{D}\right\|_{L^{p}(\Omega)}, \quad i=1, \ldots, n+1,
$$

and choosing $\epsilon$ in (30) sufficiently small the inequality (31) can be fulfilled. 
Secondly, we define the set

$$
Q_{2}=\left\{w=\left(z^{D}, g, f\right) \in Y_{p} \times L^{\infty}\left(\Gamma_{N}\right)^{n+2} \times L^{\infty}(\Omega): z_{n+1}^{D}<0\right\} .
$$

Obviously $Q_{1} \subset Q_{2}$. The following considerations need some deeper insight into the structure of the underlying reaction system. We introduce the stoichiometric subspace

$$
\mathcal{S}=\operatorname{span}\{\alpha-\beta:(\alpha, \beta) \in \mathcal{R}\} \subset \mathbb{R}^{n}
$$

and its orthogonal complement $\mathcal{S}^{\perp}, \mathbb{R}^{n}=\mathcal{S} \oplus \mathcal{S}^{\perp}$. The corresponding projection operators are denoted by $\Pi_{\mathcal{S}}: \mathbb{R}^{n} \rightarrow \mathcal{S}$ and $\Pi_{\mathcal{S}^{\perp}}: \mathbb{R}^{n} \rightarrow \mathcal{S}^{\perp}$. We show that there is a constant $c>0$ such that

$$
\left\|\lambda-\Pi_{\mathcal{S}^{\perp}} \lambda\right\|_{\mathbb{R}^{n}}=\left\|\Pi_{\mathcal{S}} \lambda\right\|_{\mathbb{R}^{n}} \leq c \sum_{(\alpha, \beta) \in \mathcal{R}}|(\alpha-\beta) \cdot \lambda| \quad \forall \lambda \in \mathbb{R}^{n} .
$$

It suffices to prove this inequality for $\lambda \in \mathcal{S},\|\lambda\|_{\mathbb{R}^{n}}=1$. If (32) is not fulfilled, then there exists a sequence $\lambda_{m}$ with $\left\|\lambda_{m}\right\|_{\mathbb{R}^{n}}=1, \lambda_{m} \in \mathcal{S}$ and $\left|(\alpha-\beta) \cdot \lambda_{m}\right| \rightarrow 0$ for all $(\alpha, \beta) \in \mathcal{R}$. We may assume that $\lambda_{m} \rightarrow \lambda_{0}$. Then $\left\|\lambda_{0}\right\|_{\mathbb{R}^{n}}=1$ and $\lambda_{0} \in \mathcal{S} \cap \mathcal{S}^{\perp}=\{0\}$, which gives a contradiction.

Corollary 2. We assume (A1)-(A5). Let $w=\left(z^{D}, g, f\right) \in Q_{2}$ be given. Then there are constants $q \in(2, p], \tau>1, \epsilon>0$ such that the following assertions hold: If

$$
\begin{aligned}
& \left\|\nabla z_{i}^{D}\right\|_{L^{p}(\Omega)}<\epsilon, \quad i=1, \ldots, n+1, \\
& \left\|\sum_{i=1}^{n}\left(\alpha_{i}-\beta_{i}\right) z_{i}^{D}\right\|_{L^{1}\left(\Gamma_{D}\right)}<\epsilon \quad \forall(\alpha, \beta) \in \mathcal{R}, \\
& \left\|g_{i}\right\|_{L^{\infty}\left(\Gamma_{N}\right)} \leq \epsilon, \quad i=1, \ldots, n+1,
\end{aligned}
$$

then there exists a $Z \in X_{q}$ such that $(q, \tau, Z, w)$ is a solution of $(\mathrm{P})$. This solution lies in a neighbourhood of an equilibrium solution $\left(q, \tau, Z^{*}, w^{*}\right)$ of $(\mathrm{P})$, and in this neighbourhood there are no solutions $(q, \tau, \widetilde{Z}, w)$ with $\widetilde{Z} \neq Z$.

Proof. Let $w=\left(z^{D}, g, f\right) \in Q_{2}$ be given. We define

$$
\begin{aligned}
& \bar{z}_{i}^{D}=\frac{1}{\left|\Gamma_{D}\right|} \int_{\Gamma_{D}} z_{i}^{D} \mathrm{~d} \Gamma, i=1, \ldots, n+1, \quad \lambda=\left(\bar{z}_{1}^{D}, \ldots, \bar{z}_{n}^{D}\right), \\
& \left(z_{1}^{D *}, \ldots, z_{n}^{D *}\right)=\Pi_{\mathcal{S}^{\perp}} \lambda, \quad z_{n+1}^{D *}=\bar{z}_{n+1}^{D}, \quad z_{n+2}^{D *}=z_{n+2}^{D}, \\
& w^{*}=\left(z^{D *},\left(0, \ldots, 0, g_{n+2}\right), f\right)
\end{aligned}
$$

and find again that $w^{*} \in Q$. Let $\left(q_{0}, \tau, Z^{*}, w^{*}\right)$ be the equilibrium solution of $(\mathrm{P})$. Because of Theorem 2 there are constants $q \in\left(2, q_{0}\right], \epsilon^{\prime}>0$ such that the equation $F_{q, \tau}(Z, w)=0$ has a locally unique solution $Z \in X_{q}$ if

$$
\left\|w-w^{*}\right\|_{Y_{p} \times L^{\infty}\left(\Gamma_{N}\right)^{n+2} \times L^{\infty}(\Omega)}=\sum_{i=1}^{n+1}\left\{\left\|z_{i}^{D}-z_{i}^{D *}\right\|_{W^{1, p}(\Omega)}+\left\|g_{i}\right\|_{L^{\infty}\left(\Gamma_{N}\right)}\right\}<\epsilon^{\prime} .
$$

From the Friedrichs inequality and the inequality (32) it follows that

$$
\begin{aligned}
& \left\|w-w^{*}\right\|_{Y_{p} \times L^{\infty}\left(\Gamma_{N}\right)^{n+2} \times L^{\infty}(\Omega)} \\
& \leq c\left(\sum_{i=1}^{n+1}\left\{\left\|\nabla z_{i}^{D}\right\|_{L^{p}(\Omega)}+\left\|g_{i}\right\|_{L^{\infty}\left(\Gamma_{N}\right)}\right\}+\sum_{(\alpha, \beta) \in \mathcal{R}}\left\|\sum_{i=1}^{n}\left(\alpha_{i}-\beta_{i}\right) z_{i}^{D}\right\|_{L^{1}\left(\Gamma_{D}\right)}\right),
\end{aligned}
$$

and $\epsilon$ in (33) can be chosen such that (34) is fulfilled. 
REMARK 3. The assertions of Corollary 2 can be interpreted as follows. Let the source terms for the Poisson equation (i.e. $f, z_{n+2}^{D}, g_{n+2}$ ) be given. Then the stationary energy model has a solution, if the driving forces for the fluxes induced by the boundary data (i.e. the gradients $\nabla z_{1}^{D}, \ldots, \nabla z_{n+1}^{D}$ ), the driving forces for all reactions evaluated on the boundary (i.e. the affinities $\sum_{i=1}^{n}\left(\alpha_{i}-\beta_{i}\right) z_{i}^{D}$ on $\left.\Gamma_{D}\right)$ and the prescribed fluxes on the boundary (i.e. $g_{1}, \ldots, g_{n+1}$ on $\Gamma_{N}$ ) are small enough. This solution is locally unique. One could expect that uniqueness should be valid globally in this case. But such a result cannot be obtained by the Implicit Function Theorem.

REMARK 4. Theorem 2 gives a local existence and uniqueness result for the stationary energy model (21), (22) in two space dimensions. Note that our equations involve cross terms with respect to all species and the temperature. Griepentrog [9] considered the special model of Subsection 1.1 for three-dimensional domains, too. He assumed that in (10) there are no cross terms, i.e. $\sigma_{n p}=P_{n}=P_{p}=0$, and he replaced the conservation law for the total energy (4) by the heat flow equation (7) which reads now as

$$
-\nabla \cdot(\kappa \nabla T)=\sigma_{n}\left|\nabla \zeta_{n}\right|^{2}+\sigma_{p}\left|\nabla \zeta_{p}\right|^{2}+\left(\zeta_{n}+\zeta_{p}\right) R
$$

Using the Implicit Function Theorem in a scale of Sobolev-Campanato spaces he obtained a local existence and uniqueness result also in this case.

REMARK 5. There are other kinds of energy models where the temperature does not mean the lattice temperature, but the carrier temperature. Such a model is studied in [2], for example. The model equations have the form (21), (22) with an additional source term in the $(n+1)$-th equation which relaxes the carrier temperature to the given constant lattice temperature. For $d$-dimensional domains, $d \geq 1$, a global existence result was derived, but under restrictive assumptions which are not fulfilled for our models. For example, the matrix $a_{i k}$ was supposed to be uniformly positive definite on $\Omega \times \mathbb{R}^{n+2}$ in contrast to our assumption (23). For two-dimensional domains a uniqueness result was obtained if the boundary data are near a thermodynamic equilibrium, but here all reactions were omitted.

REMARK 6. If in the energy model (16), (20) the temperature is considered as a constant positive parameter and the $(n+1)$-th equation is omitted, then the remaining equations form an electro-reaction-diffusion system. We studied such problems and corresponding non-stationary problems in $[3,4,5,6]$. There the boundary conditions for the continuity equations differ from those used in the present paper. But they guarantee that a stationary solution of the electro-reaction-diffusion system is a thermodynamic equilibrium, too. Especially, results concerning the long-time behaviour of solutions of the non-stationary problem were obtained. In semiconductor technology modelling so called pair diffusion models play an important role. These models are electro-reaction-diffusion systems with a nonlinear Poisson equation (since the charge numbers $q_{i}$ now depend on the potential $\varphi)$ which we investigated in $[7,8,13]$.

\section{References}

[1] G. Albinus, H. Gajewski, and R. Hünlich, Thermodynamic design of energy models of semiconductor devices, Nonlinearity 15 (2002), 367-383. 
[2] P. Degond, S. Génieys, and A. Jüngel, A steady-state system in nonequilibrium thermodynamics including thermal and electrical effects, Math. Meth. Appl. Sci. 21 (1998), 1399-1413.

[3] A. Glitzky, Elektro-Reaktions-Diffusionssysteme mit nichtglatten Daten, Habilitationsschrift, Humboldt-Universität zu Berlin, 2001, Logos-Verlag, Berlin, 2002.

[4] A. Glitzky, K. Gröger, and R. Hünlich, Free energy and dissipation rate for reaction diffusion processes of electrically charged species, Applicable Analysis 60 (1996), 201-217.

[5] A. Glitzky and R. Hünlich, Energy estimates and asymptotics for electro-reactiondiffusion systems, Z. Angew. Math. Mech. 77 (1997), 823-832.

[6] A. Glitzky and R. Hünlich, Global estimates and asymptotics for electro-reaction-diffusion systems in heterostructures, Applicable Analysis 66 (1997), 205-226.

[7] A. Glitzky and R. Hünlich, Global properties of pair diffusion models, Adv. Math. Sci. Appl. 11 (2001), 293-321.

[8] A. Glitzky and R. Hünlich, Global existence result for pair diffusion models, Preprint 784, Weierstraß-Institut für Angewandte Analysis und Stochastik, Berlin, 2002, to appear in SIAM Journal on Mathematical Analysis.

[9] J. A. Griepentrog, An application of the implicit function theorem to an energy model of the semiconductor theory, Z. Angew. Math. Mech. 79 (1999), 43-51.

[10] K. Gröger, $A W^{1, p}$-estimate for solutions to mixed boundary value problems for second order elliptic differential equations, Math. Ann. 283 (1989), 679-687.

[11] R. Haase, Thermodynamics of Irreversible Processes, Dover, New York, 1990.

[12] R. Hünlich, G. Albinus, H. Gajewski, A. Glitzky, W. Röpke, and J. Knopke, Modelling and simulation of power devices for high-voltage integrated circuits, in: MathematicsKey Technology for the Future, W. Jäger and H.-J. Krebs (eds.), Springer, Berlin, 2003, 401-412.

[13] R. Hünlich and A. Glitzky, On energy estimates for electro-diffusion equations arising in semiconductor technology, in: Partial Differential Equations. Theory and Numerical Solution, W. Jäger et al. (eds.), Research Notes in Mathematics 406, Chapman \& Hall/CRC, Boca Raton, 2000, 158-174.

[14] D. E. Kane and R. M. Swanson, Modeling of electron-hole scattering in semiconductor devices, IEEE Trans. ED 34 (1993), 120-140.

[15] L. Recke, Applications of the Implicit Function Theorem to quasi-linear elliptic boundary value problems with non-smooth data, Comm. Partial Differential Equations 20 (1995), $1457-1479$.

[16] W. V. van Roosbroeck, Theory of flow of electrons and holes in germanium and other semiconductors, Bell Syst. Techn. J. 29 (1950), 560-607.

[17] N. S. Trudinger, On imbeddings into Orlicz spaces and some applications, J. Mathematics and Mechanics 17 (1967), 473-483.

[18] G. Wachutka, Rigorous thermodynamic treatment of heat generation and conduction in semiconductor device modelling, IEEE Trans. CAD 9 (1990), 1141-1149. 